\title{
Aporte de los adhesivos dentales en la rehabilitación del desdentado. Revisión narrativa.
}

\section{Contribution of dental adhesives in the rehabilitation of the edentulous patient. Narrative review.}

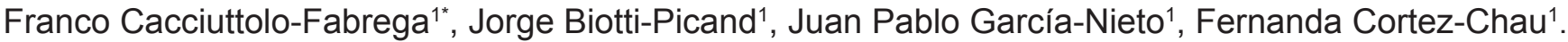

\author{
1. Facultad de Odontología Universidad de los \\ Andes. Santiago de Chile. \\ * Correspondencia autor. Dr. Franco Cacciuttolo- \\ Fabrega.|E-mail: fcacciuttolo@uandes.cl \\ Trabajo recibido el 29/07/2019. \\ Aprobado para su publicación el 17/10/2019.
}

\begin{abstract}
RESUMEN
El uso de medios auxiliares como es el caso de los adhesivos dentales permitirá que la adhesión entre la prótesis y la mucosa sea mejor y se logre una mejor retención, estabilidad y eficiencia masticatoria.

Se realizó una búsqueda en internet en las siguientes bases de datos, Pubmed, EBSCO, $B E I C$, Cochrane y Epistemonikos. Para la búsqueda electrónica se utilizaron las palabras "denture adhesive", "dental adhesive", "complete denture wearers", "retention", "support", "stability", "masticatory performance", "Candida albicans" y "quality of life" relacionadas con el operador booleano AND. Al aplicar los criterios de inclusión y exclusión terminando con 14 artículos. Finalmente, quedaron 5 ensayos clínicos aleatorizados, 3 ensayos clínicos no aleatorizados, 2 estudios observacionales de cohorte prospectivos, 3 artículos in vivo y 1 estudio in vitro. Los adhesivos dentales han demostrado un mejoramiento en la retención, adhesión, estabilidad y eficiencia masticatoria en los portadores de prótesis totales, independiente de su presentación, sea en forma de polvo, crema, tiras o almohadillas. Por medio de su uso, se ha demostrado que los portadores de prótesis totales han podido mejorar su calidad de vida, tanto en la seguridad del manejo de su aparato protésico como en el aspecto nutricional, pues permite que se alimenten de mejor forma, esto incluso es posible observar cuando se prescriben en aquellos pacientes con prótesis recién instaladas.
\end{abstract}

PALABRAS CLAVE:

Prótesis totales; Adhesivos; Retención; Estabilidad; Eficiencia masticatoria; Cándida albicans; Estomatitis subprotésica.

Rev. Clin. Periodoncia Implantol. Rehabil. Oral Vol. 12(3); 135-139, 2019.

\begin{abstract}
Introduction: When performing the oral rehabilitation of edentulous patients using total prostheses, in some cases, a slight maladjustment of the prosthetic device within the oral cavity can compromise the chewing, swallowing and phonation functions. With the use of ancillary means such as dental adhesives, which allow the adhesion between the prosthesis and the mucosa, a better retention, stability and masticatory efficiency is achieved, and therefore, an increase in the quality of life of the patient. Main objective: To perform a narrative review to describe the contribution of the use of dental adhesives in patients with total dentures. Method: We searched the following databases, Pubmed, EBSCO, BEIC, Cochrane and Epistemonikos. The words "denture adhesive", "dental adhesive", "complete denture wearers", "retention", "support", "stability", "masticatory performance", "Candida albicans" and "quality of life " were searched electronically with the Boolean operator AND. Results: As a result, 394 articles were obtained, 85 of which were selected by title, of which the repeated articles were eliminated, leaving 32 ; then, the abstracts were read, leaving 22 articles. The remaining articles were read completely, the inclusion and exclusion criteria were applied, ending with 14 articles. Finally, 5 randomized clinical trials, 3 non-randomized clinical trials, 2 prospective cohort observational studies, 3 in vivo articles and 1 in vitro study were included. Conclusion: Dental adhesives have showed an increase in retention, stability, ability and masticatory efficiency in patients with total prostheses, regardless of their presentation. Through its use, it has been demonstrated that total denture wearers have been able to improve their quality of life, even patients with recently installed prostheses.
\end{abstract}

KEYWORDS:

Dentures; Adhesive; Retention; Stability; Chewing efficiency; Stomatitis subprosthesis.

Rev. Clin. Periodoncia Implantol. Rehabil. Oral Vol. 12(3); 135-139, 2019. 


\section{INTRODUCCIÓN}

A pesar de los avances de la Odontología, es frecuente encontrar pacientes desdentados totales en nuestra población, lo que hoy en día correspondería a un $30 \%$ del total de la población de individuos mayores de 65 años ${ }^{(1-4)}$. La rehabilitación oral con prótesis total removible puede presentar ligeros desajustes del aparato protético dentro de la cavidad oral después de su instalación, lo que impide una función adecuada de dichos aparatos y que puede llevar a una modificación de los hábitos dietéticos y por ende a un déficit nutricional en aquellas situaciones más extremas ${ }^{(5,6)}$. Con el uso de medios auxiliares como es el caso de los adhesivos dentales, se puede lograr una mejor retención y estabilidad del aparato protético ${ }^{(5-7)}$. Los adhesivos se presentan en forma de polvos, pastas, cremas, tiras y almohadillas, que al colocarse en la superficie interna de la prótesis y tomar esta contacto con la superficie tisular de la cavidad oral, se produce la hidratación del material adhesivo, el que adquiere una forma viscosa que determina que la interfase entre ambas superficies se oblitere y aumente de esta forma la retención del aparato protético ${ }^{(7)}$. Los adhesivos al permitir una mejor retención de los aparatos protéticos, determinarán una mejor elección de los alimentos que se ingieren ${ }^{(6,7)}$.

Por medio de esta revisión bibliográfica se describe los aportes del uso de los adhesivos dentales en pacientes que son portadores de prótesis totales y lo que dice relación a la retención, soporte, estabilidad, eficiencia masticatoria, calidad de vida y el efecto que podría tener su uso en el desarrollo de estomatitis subprotésica en la mucosa de la cavidad oral.

\section{INDICACIONES DE USO DE ADHESIVOS DENTALES}

Rebordes muy reabsorbidos.

Retención en prótesis antiguas.

Facilitar registros máxilo mandibulares.

Pacientes con tendencia a úlceras de origen inespecífico.

Mucosa con sensibilidad extrema.

Refuerzo de la autoestima.

Disminución de la secreción salival.

Incrementar la seguridad en el uso de la prótesis por primera vez.

\section{MATERIAL Y MÉTODO}

Se realizó una revisión narrativa, a partir de un análisis de la literatura publicada en bases de datos electrónicas. Se utilizaron las siguientes palabras claves "denture adhesive", "dental adhesive", "complete denture wearers", "retention", "support", "stability", "masticatory performance", "Candida albicans y "quality of life", además de los términos MeSH "denture retention" $y$ "adhesives", relacionado con el operador booleano AND. Se seleccionaron estudios en inglés, publicados hasta noviembre del año 2016. Se complementó esta investigación con una búsqueda manual en los artículos científicos, libros y revistas electrónicas suscritas por la Biblioteca de la Universidad de los Andes. Como criterios de inclusión se consideró artículos que estuviesen disponibles en texto completo en idioma inglés y español, estudios primarios clínicos en humanos; estudios observacionales, ensayos clínicos no aleatorizados, ensayos clínicos aleatorizados e in vitro. Se eliminaron artículos correspondientes a opiniones de expertos, póster, reportes de casos y artículos que no se encontraron disponibles de manera completa.

Para evaluar el nivel de evidencia y el grado de recomendación de la literatura científica publicada, se utilizó el criterio propuesto por el Centro de Medicina Basada en la Evidencia de Oxford ${ }^{(8)}$. La evaluación de la calidad de reporte se realizó mediante las listas de cotejo Consort: para ensayos clínicos aleatorizados. Trend: para ensayos clínicos no aleatorizados. Strob: para estudios observacionales de cohorte prospectivos. Arrive: para estudios in vivo en animales de experimentación. Consort modificada para estudios in vitro (Cris). Los puntajes alcanzados por cada artículo, de acuerdo con las pautas anteriormente mencionadas fueron clasificados en tres categorías: alta, moderada y baja calidad de reporte.

El riesgo de sesgo, el que da cuenta de la validez externa del estudio, fue determinado para cada artículo en particular, en base a la pauta de riesgo de sesgo de The Cochrane Collaboration. Cada estudio obtuvo una evaluación de bajo, moderado o alto riesgo de sesgo en los siguientes casos: Bajo riesgo con una puntuación de 6 . Moderado riesgo con una puntuación entre 4 y 5 . Alto riesgo con una puntuación igual o menor a 3 .

Para la evaluación de los aspectos éticos de los estudios seleccionados, se utilizó como referencia, las pautas éticas preparadas por el Consejo de Organizaciones Internacionales de las Ciencias Médicas (CIOMS) (9). Según esto se determinó evaluar la solicitud de un consentimiento informado para cada paciente participante en el estudio, la aprobación del estudio por un comité de ética y la declaración de ausencia o presencia de conflicto de intereses por parte de los autores del estudio.

\section{RESULTADOS}

La estrategia de búsqueda arrojó un total de 394 artículos. Del total, 85 fueron seleccionados al analizar los títulos, de ellos se eliminaron los 53 que estaban repetidos. Quedando 32 artículos, se analizaron los resúmenes y se eliminaron 10 artículos. Por lo tanto, se leyeron 22 artículos completos y se aplicaron los criterios de inclusión y exclusión, quedando para el análisis 14 estudios, como se observa en el siguiente flujograma (Tabla Número 1):

Tabla 1. Flujograma de selección de artículos.

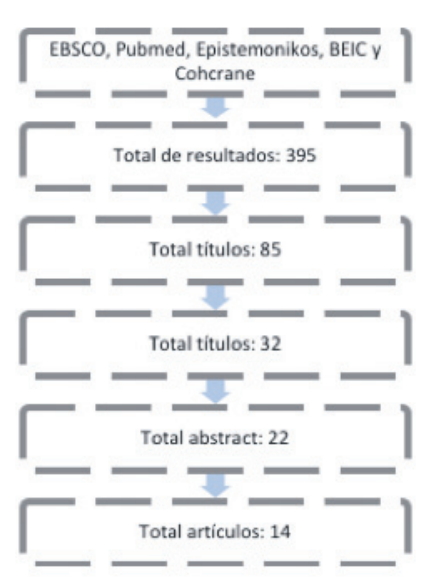

Se analizaron 9 artículos con relación a la retención y estabilidad otorgada por los adhesivos dentales, de los cuales 5 estudios eran ensayos clínicos aleatorizados, 3 ensayos clínicos no aleatorizados y 1 estudio in vivo. Sobre la relación existente entre el uso de adhesivo dental y el crecimiento del hongo Candida albicans, se encontró 2 estudios in vivo y 1 in vitro. Finalmente, con respecto al último objetivo específico correspondiente a la relación entre el uso de adhesivo dental y el aumento de la calidad de vida del paciente se encontró, 2 estudios observacionales prospectivos de cohorte (ilustración 1).

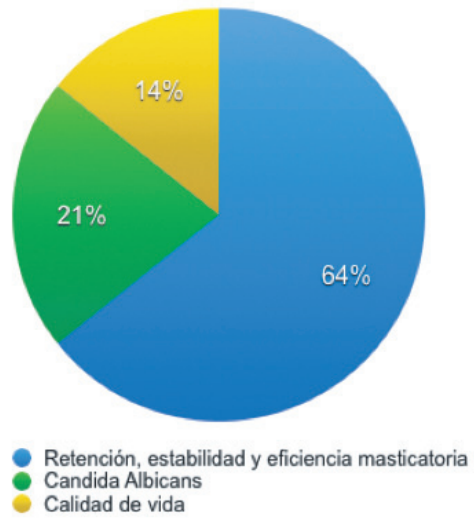

Ilustración 1. Gráfico de artículos encontrados

En el análisis del nivel de evidencia científica, un $36 \%$ presentó un nivel tipo $1 \mathrm{~b}$, el cual corresponde a un buen nivel de evidencia, conformado por los ensayos clínicos aleatorizados, un $36 \%$ presenta un nivel $2 \mathrm{~b}$, el cual corresponde a un moderado nivel de evidencia, conformado por los ensayos clínicos no aleatorizados y los estudios observacionales de cohorte prospectivo y finalmente un $29 \%$ presenta un pobre nivel de evidencia, correspondiente a 3 estudios in vivo y uno in vitro (ilustración 2).

De los 14 artículos seleccionados, 8 presentan una alta calidad de reporte, 6 moderada y ninguno presenta baja calidad de reporte (ilustración 3).

Respecto a las consideraciones éticas, un ensayo clínico aleatorizado se consideró adecuado, tres moderados y uno como inadecuado. 


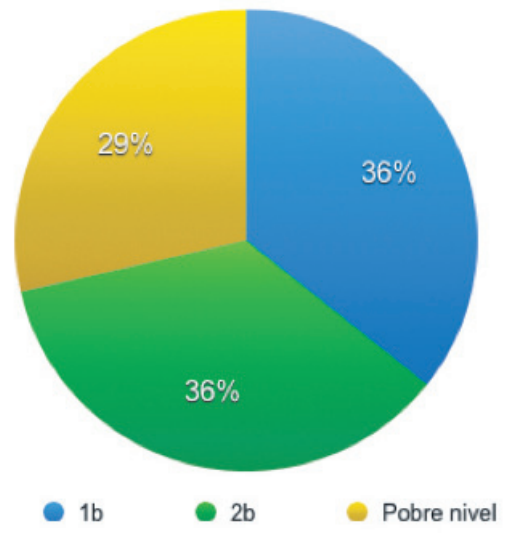

Ilustración 2. Gráfico nivel de evidencia

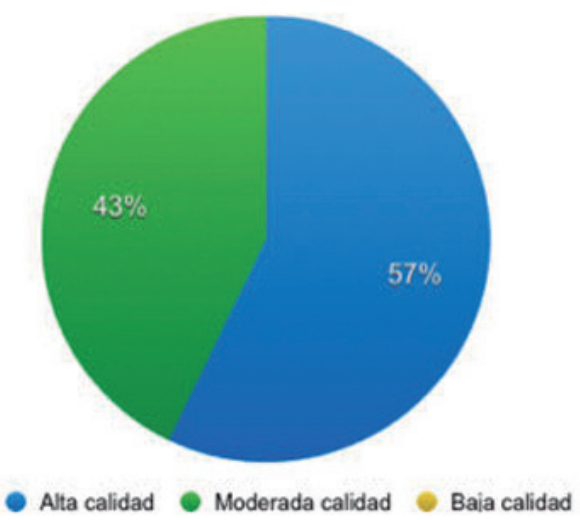

\section{Ilustración 3.}

Las consideraciones éticas de los ensayos clínicos no aleatorizados presentaron tres artículos con consideraciones éticas moderadas. En el caso de los dos estudios observacionales prospectivos de cohorte, ambos se consideraron con parámetros éticos moderados. En relación con los estudios in vivo, un artículo se consideró como moderado respecto de las consideraciones éticas y dos artículos como inadecuado. Finalmente, con respecto al estudio in vitro se consideró como inadecuado por sus consideraciones éticas (ilustración 4).

Respecto al riesgo de sesgo, cuatro artículos presentaron un alto riesgo de sesgo, cinco moderados y uno bajo riesgo de sesgo (ilustración 5).

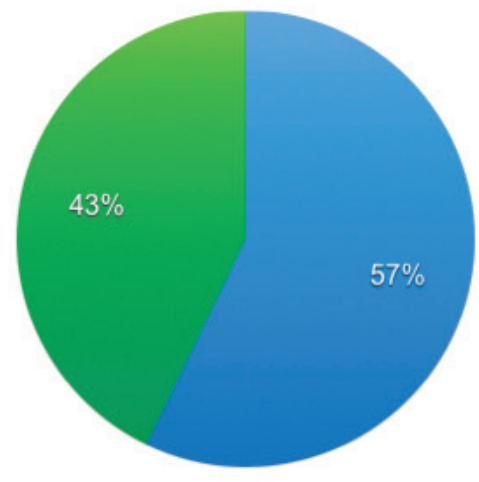

Alta calidad Moderada calidad Baja calidad

Ilustración 4. Gráfico consideraciones éticas

\section{DISCUSIÓN}

Para mejorar la retención y estabilidad de una prótesis total se puede utilizar adhesivos dentales, se ha comprobado que los beneficios del uso de estos también se obtienen en aquellas prótesis bien ajustadas al momento de su instalación y en pacientes que utilizan aparatos protéticos por primera vez ${ }^{(10)}$.

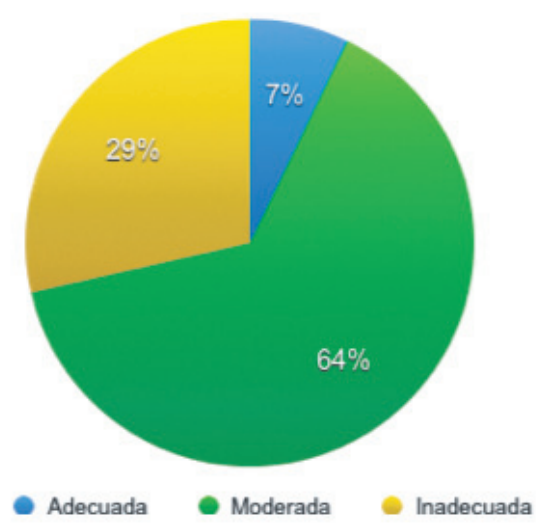

Ilustración 5. Gráfico riesgo de sesgo

Con respecto a la contribución de los adhesivos dentales en la retención, estabilidad, soporte y eficiencia masticatoria de las prótesis totales, los nueve artículos estudiados concuerdan en que estos otorgan un mejoramiento en estas propiedades.

Gonçalves et al. realizaron un estudio con un nivel de evidencia $1 \mathrm{~b}$ y un grado de recomendación $\mathrm{A}$, con el objetivo de evaluar el efecto del adhesivo en pacientes que lo usan en crema, en tiras o que no utilizan adhesivos, obtuvieron resultados donde la eficiencia masticatoria mostró diferencias estadísticamente significativas al utilizar adhesivo en crema $(p<0,001)$, al compararlo con las otras 2 presentaciones. El ciclo masticatorio fue más rápido cuando los pacientes usaban adhesivo en crema $(p>0,001)$. También se observó que el adhesivo dental en crema presentaba una mayor velocidad al masticar "maní" $(p<0,001)$ al compararlo con los adhesivos en tiras y aquellos que no utilizaron presentaron la velocidad más baja ${ }^{(10)}$. Ambas presentaciones de adhesivos (crema y tiras) demostraron mejores resultados en la eficiencia masticatoria al utilizarlos, siendo la crema, la que mejor resultados presentó, obteniendo un $36 \%$ de incremento en la eficiencia masticatoria. Por lo que se consideró, este último, como más eficiente y con un efecto más prolongado. También el adhesivo en crema permitió incrementar la velocidad de los ciclos de masticación independiente del alimento utilizado, "maní" u "optocal, " además, los adhesivos mostraron incrementar la retención y estabilidad, lo que mejora la fuerza oclusal durante la masticación, pudiendo obtener partículas más pequeñas y uniformes durante el proceso ${ }^{(10)}$, sin embargo, una limitación encontrada en este estudio es que no se evaluó las variables masticatorias cuando los pacientes usaban sus prótesis totales antiguas, todos los resultados se obtuvieron a partir de nuevas prótesis. Por lo que el uso de adhesivo dental es beneficioso en prótesis bien ajustadas y recientemente instaladas ${ }^{(10)}$.

Koronis et al realizaron un estudio con un nivel de evidencia $1 \mathrm{~b}$ y un grado de recomendación A. Reportaron más de un $40 \%$ de mejoras en la retención de prótesis totales con el uso de adhesivos, un aumento de más del $70 \%$ en la eficiencia masticatoria, y se mantuvo activo por 2 horas en más del $75 \%$ de los voluntarios estudiados, además, hubo un aumento de más del $65 \%$ en la confianza de los portadores de prótesis a la hora de relacionarse socialmente. El mejor resultado en el aumento de la retención y habilidad masticatoria, fue en aquellos pacientes que mostraron una menor retención de su prótesis sin uso de adhesivo en un comienzo del estudio ${ }^{(11)}$.

Los adhesivos tipo almohadillas han sido considerados útiles como ayuda en el funcionamiento de la prótesis en pacientes con grandes reabsorciones y a pesar de que se ha relacionado a este tipo de adhesivo con la aparición de lesiones de tipo irritativas en la mucosa, disminución de la dimensión vertical y aceleración en el proceso de reabsorción del reborde, estas aseveraciones no han sido fundamentadas a través de la evidencia científica. Se ha documentado en la literatura los efectos adversos del uso de adhesivos dentales, en especial los que tienen en su composición zinc, que pueden provocar irritabilidad, cambios de tipo neurológicos en algunos casos, por lo que se aconseja utilizar siempre adhesivos libres de zinc ${ }^{(11)}$.

Oliveira Junior et al. realizaron un estudio con un nivel de evidencia 1b y un grado de recomendación A, por medio del método del tamizaje, demostraron que el uso de adhesivo aumenta la eficiencia masticatoria, independiente del tipo de presentación. Observaron que el mecanismo de los adhesivos es aumentar la viscosidad de la saliva entre la base de la prótesis y el tejido mucoso, lo que optimiza las propiedades cohesivas 
y adhesivas, favoreciendo de esta manera, la retención, estabilidad y eficiencia masticatoria. Al usar el adhesivo se incrementa la capacidad de moler y triturar alimentos, por lo que también mejora la digestión y permite que los pacientes puedan ingerir alimentos de consistencia más fibrosa, mejorando su nutrición ${ }^{(12)}$. Polyzois et al. en su estudio de nivel de evidencia $2 \mathrm{~b}$ y con un grado de recomendación $\mathrm{B}$, concluyeron que el uso de adhesivos aumenta la resistencia a las fuerzas de desalojo y se logra mejor retención y estabilidad del aparato protético ${ }^{(13)}$.

Kalra $\mathrm{P}$ et al. realizaron un estudio con un nivel de evidencia $2 \mathrm{~b}$ y grado de recomendación $\mathrm{B}$, demostró que el uso de adhesivo mejora la eficiencia masticatoria y al compararlo con prótesis sin adhesivos hay diferencias estadísticamente significativas cuando se usa una fuerza al nivel incisal para lograr el desalojo de prótesis totales superiores. Los mejores resultados en la retención medida por incremento de la fuerza incisal, se encontraron al utilizar adhesivo en crema, seguido por la presentación en polvo y por último en tiras ${ }^{(14)}$.

Pradíes et al. realizaron un estudio con un nivel de evidencia $1 \mathrm{~b}$ y un grado de recomendación $\mathrm{A}$, observaron claramente una mejoría en la retención, estabilidad y eficiencia masticatoria de los pacientes portadores de prótesis totales, al utilizar dos tipos distintos de adhesivos dentales en crema, reportando un $70 \%$ de aumento en la retención con una duración de hasta ocho horas de permanencia en la cavidad oral, se realizó en pacientes con prótesis superior e inferior, los mejores resultados se obtuvieron en aquellos maxilares que en un comienzo presentaban mayores problemas de retención (15).

Figueiral et al. en un estudio con un nivel de evidencia $2 \mathrm{~b}$ y un grado de recomendación $B$, concluyeron que el uso de adhesivo incrementó la retención de la prótesis, a excepción del adhesivo en tiras que no arrojó diferencias estadísticamente significativas ${ }^{(16)}$

Con el objetivo de evaluar los beneficios del adhesivo en prótesis mandibulares, Mañes et al. realizaron, un estudio in vivo con un pobre nivel de evidencia y concluyeron que el uso de adhesivo dental aumenta la viscosidad de la saliva acrecentando la retención. Sin embargo, al comparar el rango de incremento en la retención de las prótesis maxilares con las mandibulares, es menor en estas últimas, ya que el área a cubrir es mucho menor en relación al maxilar superior ${ }^{(14,16)}$. Este aumento en la retención se espera que disminuya a medida que el tiempo transcurre y la saliva vaya disolviendo gradualmente el adhesivo (14), (16).

Con respecto a la relación entre el adhesivo y el incremento de la calidad de vida, se analizó un estudio de cohorte prospectivo del año 2013 , con un nivel de evidencia $2 b$ y con un grado de recomendación $\mathrm{B}$, Bartlett et al. observaron incrementos estadísticamente significativos del consumo de frutas, vegetales y vitamina $c$ por día $(p<0,0001)$, por otra parte, hubo una disminución significativa en la ingesta de grasas saturadas $(p<0,0001)$, al igual se reveló aumentos estadísticamente significativos, en la habilidad para morder $(p=0,0175)$ y masticar $(p=0.0074)$, permitiendo un mejoramiento de la calidad nutricional y por ende una mejor calidad de vida ${ }^{(5)}$.

A través de un cuestionario llamado GOHAl Nicolas et al. en un estudio observacional de cohorte prospectivo con un nivel de evidencia $2 \mathrm{~b}$ y un grado de recomendación $\mathrm{B}$, realizaron un seguimiento durante seis meses, concluyeron que se produce un significativo mejoramiento en la calidad de vida de los pacientes portadores de prótesis totales al usar el adhesivo, lo que se vio reflejado en los puntajes de GOHAI ( $F=12$, $\mathrm{p}<0,001)^{(6)}$.

Nicolas et al. observaron, que el uso de adhesivo en los tres meses después de instalado el aparato protético, se vio facilitada la integración psicosocial y un mejoramiento del accionar neuromuscular en los portadores de prótesis totales, mejorando la eficiencia masticatoria, el confort y seguridad en el uso de sus aparatos protésicos ${ }^{(6)}$.

En cuanto a la evaluación de la relación que existe entre el adhesivo dental y la Candida albicans, en un estudio in vitro con un pobre nivel de evidencia, Sampaio-Maia et al. concluyeron que dos adhesivos dentales tenían un efecto inhibitorio sobre la Candida albicans; Kukident y Corega, ambos con sus presentaciones en crema ${ }^{(17)}$. Kukident presentó un marcado efecto inhibitorio sobre el crecimiento de la Candida, alcanzando un $95 \%$ de inhibición. Mientras que el Corega tuvo un efecto más moderado, reduciendo su crecimiento en un $37 \%$. El efecto inhibitorio en el crecimiento de la Candida con el uso del adhesivo Kukident mostró ser dependiente de su concentración, a diferencia del Corega donde su efecto no dependía de su concentración. Adicionalmente se observó que la mayoría de los adhesivos inducen cambios morfológicos en las células fúngicas y sus colonias, tanto a nivel microscópico como macroscópico, determinando que el desarrollo del hongo sea más difícil y por ende que se manifieste en la mucosa subyacente ${ }^{(17)}$.

Finalmente, como información complementaria, Sampaio-Maia et al. también quisieron evaluar la contaminación microbiana del adhesivo dental. Donde solo aquellos adhesivos en presentación de tipo polvo, mostraron crecimiento microbiano, lo que concuerda con un estudio realizado anteriormente que encontraron hongos y bacterias en 4 adhesivos dentales en polvo (18). Aunque no se identificaron microorganismos patógenos potencialmente dañinos, esta realidad debería ser considerada en especial en aquellos individuos inmunocomprometidos, los valores más bajos de contaminación lo obtuvieron aquellos adhesivos en presentación de crema, lo que puede estar relacionado a que presentan agentes antimicrobianos en su composición tales como; borato de sodio, tetraborato de sodio, hexaclorofeno, benzoato de propil hidroxi y etanol $(16,17)$. Sin embargo, una importante limitación en este estudio es que, al ser in vitro, las observaciones no son siempre reproducibles in vivo con los componentes de la saliva y flujo salival, también el $\mathrm{pH}$ intraoral puede interferir en el crecimiento de la Candida. Otros factores como trauma, inflamación y la presencia adicional de condiciones patológicas, también deben ser considerados ${ }^{(18)}$.

Oliveira et al. realizaron, un estudio in vivo con un pobre nivel de evidencia, con el objetivo de evaluar el efecto del uso de adhesivo dental de tipo cinta o tira en la colonización de Candida albicans y otras especies en pacientes completamente edéntulos por un período de 14 días. Los fabricantes de los adhesivos utilizados en este estudio afirman que el etanol presente en la composición del producto tiene un efecto antifúngico que podría no haberse notado durante el estudio ya que los dos grupos observados no presentaron diferencias significativas entre ellos, se observó que el uso de Ultra Corega en tira, por un período de 14 días, no causó un significativo aumento ni disminución en el recuento de Candida y tampoco en otros tipos de hongos presentes en la cavidad oral de los individuos estudiados ${ }^{(19)}$.

Oliveira et al. encontraron, que los adhesivos no tienen un efecto positivo en el aumento de Candida, concordando de esta manera con otros estudios, sin embargo, debe ser considerado el hecho de que todos los individuos en este estudio usaron prótesis totales nuevas, bien adaptadas a la mucosa y con una adecuada retención y estabilidad, en oposición a lo que ocurre con la mayoría de los individuos que usan adhesivos, ya que intentan con su uso mejorar la retención y estabilidad en prótesis antiguas. También se le entregó a los pacientes consejos de higiene oral y un correcto uso del adhesivo ${ }^{(19)}$. Respecto de estos tópicos, es necesario más estudios y por períodos más largos de observación.

En el año 2011, los autores Ozkan et al. realizaron un estudio in vivo, con un pobre nivel de evidencia, evaluaron el efecto del adhesivo dental sobre los microorganismos por un período de 2 meses, analizaron si es que existía un mayor riesgo al utilizar el adhesivo por un período prolongado, este estudio es considerado como el primero en evaluar un espectro amplio de microorganismos encontrados en la flora oral. Los Cocos gram positivos son las bacterias aisladas más predominantes en un rango de un $60 \%$ a $70 \%$ de la composición de la placa total, se encontraron tanto en la mucosa del paladar como en la superficie interna de la prótesis ${ }^{(20)}$.

Al especificar las distintas especies de microorganismos se revelaron Streptococos, incluyendo S. molleri, S. mutans, S. salivarius como también $\mathrm{S}$. aureus. Este último siendo el microorganismo más predominante en la mucosa del paladar, superficie de la prótesis y en muestras de saliva, demostrándose que no habrá un aumento de los microorganismos presentes en la cavidad oral (20).

En un estudio de dos semanas de Oliveira et al. se demostró que no se producía un aumento o disminución de microorganismos en la cavidad oral, al utilizar adhesivo en crema, por lo que esta investigación previa concuerda con los resultados del estudio de Ozkan et al (20).

En la literatura, no existe reportes de caso con respecto a adhesivos que produzca efectos dañinos en los tejidos blandos o alteración en la salud general de los pacientes que lo utilicen por un período extendido, incluso después de 2 meses de uso ${ }^{(20)}$.

\section{CONCLUSIÓN}

Los estudios demuestran que la retención de las prótesis totales se ve incrementada con el uso de adhesivo dental en la forma de crema, permitiendo que esta se mantenga por alrededor de cuatro a seis horas.

La retención y estabilidad de la prótesis cumplen un rol fundamental en la eficiencia masticatoria. El aumento de la retención de la prótesis que se logra con el uso de adhesivo representa un factor clave en el éxito del tratamiento rehabilitador y en la calidad de vida del paciente, tanto en prótesis bien ajustadas como en las que tienen un mayor desajuste.

Los adhesivos dentales aumentan la eficiencia masticatoria en los portadores de prótesis totales, independiente de si su presentación es en crema, pasta, polvo, almohadilla y tira. El aumento en la retención se puede observar en prótesis maxilares y mandibulares, sin embargo, en estas últimas el incremento es menor, las prótesis mandibulares se caracterizan por presentar los mayores problemas de retención por el 
área de superficie que cubren.

Se ha observado un mayor aumento de la retención, eficiencia masticatoria, autoconfianza al relacionarse con el resto y satisfacción con la prótesis, en aquellos pacientes que presentan mayores problemas de retención antes de utilizar el adhesivo como medio auxiliar de retención del aparato protético.

La combinación entre el uso de adhesivo dental y consejos para seguir una dieta saludable logra mejoras significativas en la elección de la alimentación de los portadores de prótesis. El uso de adhesivos mejora la salud oral, relacionándose así, directamente con una mejor calidad nutricional y de vida.

La mayoría de los adhesivos dentales estudiados inducen severos cambios morfológicos en las células fúngicas y sus colonias, tanto macroscópicos como microscópicos. Kukident y Corega, ambos adhesivos en presentación de crema, tienen un efecto inhibitorio sobre el desarrollo de la Candida albicans en la cavidad oral.

No existen diferencias significativas en cuanto al recuento de microorganismos presentes en pacientes que utilizan adhesivo dental y los que no. El uso prolongado de adhesivo, en un período de más de dos meses, no produce un incremento en el número de microorganismos de la flora oral.

Hoy en día en base a lo anterior, se puede sugerir que el uso de adhesivos dentales en pacientes portadores de prótesis por primero vez de, será muy beneficioso para lograr una mejor adaptabilidad, retención y eficiencia masticatoria, durante el período inicial de pos instalación.

\section{CONFLICTO DE INTERESES}

Los autores no declaran conflicto de intereses.

\section{Bibliografía}

1. Méndez Silva JE, Madrid Troconis CC, Tirado Amador LR. Saliva and alternative adhesive systems for complete dentures. Rev Fac Odontol Univ Antioquia. 2013 Dec;25(1):208-18.

2. Thomson WM. Social inequality in oral health. Community Dent Oral Epidemiol. 2012 Oct;40 Suppl 2:28-32.

3. Gaio EJ, Haas AN, Carrard VC, Oppermann RV, Albandar J, Susin C. Oral health status in elders from South Brazil: a population-based study: Oral health in older Brazilians. Gerodontology. 2012 Sep;29(3):214-23.

4. Chestnutt IG, Binnie VI, Taylor MM. Reasons for tooth extraction in Scotland. J Dent. 2000 May;28(4):295-7.

5. Bartlett DW, Maggio B, Targett D, Fenlon MR, Thomas J. A preliminary investigation into the use of denture adhesives combined with dietary advice to improve diets in complete denture wearers. J Dent. 2013 Feb;41(2):143-7.

6. Nicolas E, Veyrune J, Lassauzay C. A six-month assessment of oral health related quality of life of complete denture wearers using denture adhesive: a pilot study. J Prosthodont. 2010 Aug;19(6):443-8.

7. Adisman IK. The use of denture adhesives as an aid to denture treatment. $J$ Prosthet Dent. 1989 Dec;62(6):711-5

8. Oxford Centre for Evidence-based Medicine - Levels of Evidence (March 2009) [Internet]. CEBM. 2009 [cited 2016 Nov 16]. Available from: http://www.cebm.net/ oxford-centre-evidence-based-medicine-levels-evidence-march-2009/

9. Consejo de Organizaciones Internacionales de las Ciencias Médicas. Pautas éticas internacionales [Internet]. [cited 2016 Nov 13]. Available from: http://www. cioms.ch/publications/guidelines/pautas eticas internacionales.htm

10. Medina-Solís CE, Pérez-Núñez $\bar{R}$, Maupomé $G$, Casanova-Rosado JF. Edentulism among Mexican adults aged 35 years and older and associated factors. Am J Public Health. 2006 Sep;96(9):1578-81.

10. de Oliveira Junior NM, Rodriguez LS, Mendoza Marin DO, Paleari AG, Pero $A C$, Compagnoni MA. Masticatory performance of complete denture wearers after using two adhesives: a crossover randomized clinical trial. J Prosthet Dent. 2014 Nov;112(5):1182-7.
11. Polyzois G, Lagouvardos P, Frangou M, Stefaniotis T. Efficacy of denture adhesives in maxillary dentures using gnathodynamometry: a comparative study. Odontology. $2011 \mathrm{Jul} ; 99(2): 155-61$

12. Pradíes G, Sanz I, Evans O, Martnez F, Sanz M. Clinical study comparing the efficacy of two denture adhesives in complete denture patients. Int J Prosthodont. 2009 Aug:22(4):361-7.

13. Hobrink J, Zarb GA, Bolender CL, Eckert $S$, Jacob $R$, Fenton $A$, et al Prosthodontic treatment for edentulous patients: complete dentures and implantsupported prostheses. Elsevier : 2003. 1763 p.

14. Mañes JF, Selva EJ, De-Barutell A, Bouazza K. Comparison of the retention strengths of three complete denture adhesives: an in vivo study. Med Oral Patol Oral Cirugia Bucal. 2011 Jan;16(1): e132-136

15. Kalra P, Nadiger R, Shah FK. An investigation into the effect of denture adhesives on incisal bite force of complete denture wearers using pressure transducers - a clinical study. J Adv Prosthodont. 2012 May;4(2):97-102.

16. Figueiral MH, Fonseca PA, Pereira-Leite C, Scully C. The effect of different adhesive materials on retention of maxillary complete dentures. Int J Prosthodont. 2011 Apr;24(2):175-7.

17. Goiato MC, Ribeiro PDP, Garcia AR, dos Santos DM. Complete denture masticatory efficiency: a literature review. J Calif Dent Assoc. 2008 Sep;36(9):683-6. 18. Sheiham A, Steele JG, Marcenes W, Lowe C, Finch S, Bates CJ, et al. The relationship among dental status, nutrient intake, and nutritional status in older people. J Dent Res. 2001 Feb;80(2):408-13

19. Nowjack-Raymer RE, Sheiham A. Association of edentulism and diet and nutrition in US adults. J Dent Res. 2003 Feb;82(2):123-6.

20. Arendorf TM, Walker DM. The prevalence and intra-oral distribution of Candida albicans in man. Arch Oral Biol. 1980;25(1):1-10. 